\title{
Introducir la indagación en el aula en busca de investigación científica
}

\author{
Katerin Farieta Bonilla ${ }^{5}$ \\ katefarieta@gmail.com
}

\section{Resumen}

Partiendo de la poca motivación e interés de los estudiantes por la investigación científica, este proyecto pretende implementar la indagación como una herramienta de aprendizaje fundamental para desarrollar la creatividad de los estudiantes. Para dicho propósito se diseñó una propuesta metodológica basada en el modelo de aprendizaje por investigación, sintetizada por Gil Pérez D. en su artículo Contribución de la historia y de la filosofía de las ciencias al desarrollo de un modelo de enseñanza-aprendizaje como investigación, aplicada a una muestra de estudiantes, y que permite concluir que los procesos de experimentación influyen positivamente en los procesos de aprendizaje y de desarrollo de actitud científica en los estudiantes.

\section{Palabras clave}

Investigación científica, indagación, escepticismo, informes de laboratorio y motivación.

5. Estudiante del Departamento de Química - UPN 


\section{Introducción}

Las cifras estadísticas que expresan el estado del desarrollo científico en Colombia son alarmantes. A nivel latinoamericano, nuestro país se encuentra tan solo por encima de Perú con 101 científicos por millón de habitantes y ni hablar a nivel internacional, teniendo en cuenta que países como Japón cuentan con 5053 científicos por millón de habitantes.

En efecto, en las aulas de clase es evidente la ausencia de una de las principales herramientas del conocimiento y, como se defiende a lo largo de este artículo, de la investigación científica: la indagación. La ausencia de la pregunta se ve en situaciones como el temor de los alumnos para cuestionar acerca de los conceptos enseñados por el maestro y, lo que es más alarmante, la indiferencia de los maestros mismos hacia las preguntas y dudas de los estudiantes, ya sea porque no conocen las respuestas o porque simplemente consideran que son irrelevantes. Todo esto se constituye como un obstáculo para la creatividad de los jóvenes y, por lo tanto, para las ciencias y su enseñanza.

Es evidente la necesidad de que la educación tome en serio su papel como formadora de futuros hombres de ciencia. Para tal fin, se propone despertar el escepticismo para que el estudiante adopte una posición crítica basada en la duda y en la formulación de hipótesis. Se señala que la tierra era plana, esto fue obvio en un tiempo. Fue obvio que los cuerpos pesados caían más de prisa que los ligeros. Fue obvio que algunas personas eran esclavas por naturaleza y por decreto divino. Fue obvio que las sanguijuelas curaban la mayoría de las enfermedades. Fue obvio que hubo un sistema de referencia en reposo absoluto. Fue obvio que existía un lugar que ocupaba el centro del universo y que la tierra se encontraba en este lugar privilegiado (Sagan, 1995). Así pues, el problema que ocupa las páginas siguientes gira en torno a cómo se puede desarrollar la creatividad de los estudiantes y despertar su interés por la investigación científica a partir de la indagación.

El método a seguir está basado en el artículo de Carol S. Avery Aprender cómo se investiga. Investigar cómo se aprende, donde la autora propone la pregunta como fundamento esencial de todo proceso investigativo. Refiriéndose al desarrollo histórico de las ciencias naturales, Avery plantea que una pregunta llevó a otras preguntas y a buscar respuestas que ponían en juego todos los componentes básicos del lenguaje: escuchar, hablar, escribir y leer. De esta manera, el método a seguir en las aulas debe contener por lo menos las siguientes actividades: lectura diaria (bibliografía amplia), escritura de dudas y preguntas (una especie de diario), socialización y, finalmente, la resolución de los interrogantes de los estudiantes.

\section{Referente teórico}

Introducir la indagación como una premisa básica para la enseñanza en el aula resulta importante pues, dados los problemas que se han enunciado anteriormente, es evidente la necesidad de cultivar el rigor científico y la creatividad de los estudiantes para estimular su interés por las ciencias. Lo que aquí se propone es cambiar nuestra mirada habitual de la ciencia por una visión escéptica que se pregunte por el cómo y el por qué de los conceptos. De este modo el estudiante tendrá la posibilidad de adoptar una posición crítica, basada en la duda y en la formulación de hipótesis, lo que le permitirá tener una apreciación mejor fundamentada de las leyes de la naturaleza, apartando de su medio de aprendizaje aquellas creencias erróneas que distorsionan el conocimiento del mundo y su funcionamiento.

Es claro que, usualmente, el entorno ejerce una influencia en las creencias de los estudiantes que les impide tener un conocimiento preciso 
Introducir la indagación como una premisa básica para la enseñanza en el aula resulta importante pues, dados los problemas que se han enunciado anteriormente, es evidente la necesidad de cultivar el rigor científico y la creatividad de los estudiantes para estimular su interés por las ciencias. Lo que aquí se propone es cambiar nuestra mirada habitual de la ciencia por una visión escéptica que se pregunte por el cómo y el por qué de los conceptos.

de la naturaleza y su funcionamiento y, por lo tanto, es necesario inculcarles una actitud de duda frente a todo lo que perciben intuitivamente. Este tipo de creencias impiden el desarrollo de la ciencia; el papel de toda educación que pretenda formar futuros científicos debe, en primer lugar, enfocarse en despertar la creatividad de los jóvenes para que éstos puedan deshacerse de los falsos conceptos que impiden el desarrollo de la ciencia.

Algunas de las características más notorias de los niños que se encuentran cursando los niveles educativos básicos son la creatividad y la capacidad de asombro. Estas cualidades, elementales para cualquier estudioso de las ciencias, se manifiestan en el entusiasmo de los niños para formular preguntas perspicaces, acerca de lo que perciben o alrededor de los detalles de los conceptos que les da el maestro. Carl Sagan dice que en este nivel los niños "no conocen la idea de una pregunta estúpida”, más adelante plantea que "hay preguntas ingenuas, preguntas tediosas, preguntas mal formuladas, preguntas planteadas con una inadecuada autocrítica. Pero toda pregunta es un clamor por entender el mundo. No hay preguntas estúpidas" (Sagan, 1995, p. 349).

Por otra parte, los jóvenes que cursan niveles intermedios y avanzados presentan rasgos to- talmente diferentes. Usualmente memorizan conceptos, pero generalmente han perdido la capacidad de asombro y el placer por el descubrimiento, presentes en etapas anteriores. Esta realidad se puede explicar a partir de diferentes situaciones, pero los factores determinantes parecen ser la presión que los demás estudiantes, los maestros y la sociedad ejercen sobre los alumnos interesados por la investigación científica.

De este modo, se pretende entender la investigación científica como una búsqueda metódica que se lleva a cabo pasando de una pregunta a otra. Carol S. Avery expresa su experiencia como investigadora de la siguiente manera: "Cada investigación se inició con una pregunta que yo tenía la necesidad de explorar. Esta pregunta llevó a otras preguntas y a buscar respuestas que ponían en juego todos los componentes básicos del lenguaje: escuchar, hablar, escribir y leer" (Avery, 1990, p. 52). Se propone entonces en este proyecto una estrategia para que las clases de ciencias se lleven a cabo de tal forma que los estudiantes se familiaricen y se interesen por la investigación científica.

En primer lugar, se debe solicitar a cada estudiante que haga una lectura previa acerca del tema correspondiente a la clase del día; el docente recomendará una bibliografía suficientemente amplia con el propósito de que cada estudiante consulte una fuente distinta. De este modo, cada estudiante contará con una visión diferente del tema $y$, por tanto, surgirán dudas y preguntas de diferente clase. Además, cada estudiante llegará a la clase con sus preguntas y dudas escritas; a partir de esto, el maestro los orientará para que puedan resolver sus dudas por sí mismos. Las respuestas de las preguntas serán construidas a partir de la socialización, la experimentación y todas las ayudas académicas que se puedan ofrecer al estudiante para que, a partir de su creatividad, descubra nuevos conceptos que satisfagan 
sus inquietudes. Es importante motivar al estudiante a que se fije en cada uno de los aspectos de su trabajo y del trabajo de los demás, ya que, gracias a esta labor, surgirán nuevas dudas y preguntas. Así, el trabajo individual de los estudiantes se compensará y enriquecerá con la experiencia grupal.

Por último, el maestro deberá aclarar y complementar los conceptos adquiridos por los estudiantes a lo largo del proceso. Así, ayudará al estudiante a que investigue de forma creativa y, al mismo tiempo, evitará que este último se haga ideas incompletas o erróneas. A partir de las preguntas hechas por los estudiantes, el docente podrá realizar una selección de las preguntas más apropiadas para emprender la investigación y se encargará de que los estudiantes continúen con el proceso de indagación. Los resultados obtenidos se tomarán como un elemento a evaluar.

\section{Objetivos generales}

- Implantar la indagación como una herramienta de aprendizaje fundamental para desarroIlar la creatividad de los estudiantes y para la investigación científica.

- Despertar en los estudiantes un renovado interés por la ciencia y la investigación, basado en su curiosidad y creatividad.

\section{Objetivos específicos}

- Inculcar en los estudiantes el hábito de preparar los temas previstos, dudas y preguntas antes de cada clase.

- Propiciar espacios para que los estudiantes participen activamente en clase, construyendo conocimiento a partir de sus preguntas.

- Introducir el trabajo en grupo como una posibilidad útil para que los estudiantes compartan entre sí y con el maestro, afianzando sus conocimientos, aclarando sus dudas.
- Desarrollar en los estudiantes la capacidad de juzgar los conceptos que reciben, tanto de los maestros, como del medio en que se desenvuelven fuera de las aulas, con una posición escéptica.

\section{Metodología}

La metodología de este proyecto se pretende realizar con el modelo de Aprendizaje por investigación. Dicho modelo pretende, inicialmente, ver al estudiante como investigador. Lo que se busca es que el estudiante realice una investigación dirigida y acompañada por el maestro de forma permanente.

En síntesis, el modelo de Aprendizaje por investigación propone:

1. Creación de una situación problemática (continua y a menudo confusa).

2. Construcción y fundamentación de hipótesis.

3. Elaboración de estrategias diversas, incluyendo el diseño y la realización de experimentos.

4. Interpretación de los resultados de la parte práctica, incluyendo, además, resultados de otras investigaciones.

5. Intercambio de información con otros equipos de trabajo.

6. Finalmente, el enunciado preciso del problema.

El método será evaluado con la prueba de Likert, la que mostrará el cambio de actitud de los estudiantes hacia la ciencia. Se pretende aplicar dicha prueba al inicio y al final de la parte práctica del proyecto.

Este proyecto se desarrolló en el Instituto Distrital Tomas Carrasquilla, ubicado en el barrio Simón Bolívar, con 35 estudiantes entre 18 y 19 años del grado 11. Este es un grupo que presentaba indisciplina, baja motivación hacia la química y poco conocimiento frente a la elaboración de un proyecto científico. 
Para poder llevar a cabo este proyecto se diseñaron varios instrumentos con el fin de desarrollar en los estudiantes una mejor actitud hacia la ciencia y, a la vez, poder lograr que desarrollaran un buen informe, teniendo en cuenta las observaciones que se hicieron en la primera práctica. Algunas de esas técnicas e instrumentos se describen a continuación.

1. Los estudiantes escogieron una fruta, realizaron una consulta sobre sus propiedades y las verificaron en el laboratorio.

2. Se planeó y realizó un laboratorio casero sobre la elaboración del vino y del ácido acético de la fruta previamente escogida.

3. Se solicitó a los estudiantes el avance quincenal del laboratorio.

4. Se diseñó y realizó una práctica en el laboratorio del Colegio para estudiar las propiedades físicas y químicas de los alcoholes y ácidos orgánicos.

5. Se solicitaron informes sustentados para valorar el aprendizaje de los conceptos trabajados.

6. Se aplicó la prueba de actitud hacia la ciencia, antes y después de la implementación del proyecto.

\section{Resultados - Análisis}

A continuación se analizarán los resultados obtenidos, teniendo en cuenta dos aspectos fundamentales: primero, la actitud de los estudiantes frente al proceso de aprendizaje por investigación; y, segundo, el aprendizaje de elaboración de informes por medio de ensayo y error.

\section{* Primera actividad}

De esta primera actividad se comprobó, en primera instancia, lo observado en la práctica uno. Los estudiantes entregaron un informe muy pobre, con poca bibliografía y sin ningún tipo de análisis acerca del laboratorio. El informe se devolvió con las correcciones del caso y se pidió un nuevo informe; el segundo trabajo arrojo mejores resultados, la bibliografía fue más amplia y se realizaron los análisis con un lenguaje más científico.

\section{* Segunda actividad}

En cuanto al laboratorio casero, al principio los estudiantes se mostraron interesados, ya que la idea de realizar las variables que quisieran les pareció interesante. En cuanto a los informes quincenales, les pareció algo exagerado. Alrededor del experimento realizado para la obtención del vino y del vinagre se observaron algunas consecuencias interesantes. Las reacciones físicas y químicas observadas por los estudiantes resultaron para ellos bastante llamativas, algunos observaron el estallido de las botellas, a causa del sellamiento hermético del envase; otros obtuvieron el efecto contrario, debido a que al abrir la botella para medir la temperatura el alcohol se oxidó. Se desarrolló un debate interesante, pues todos querían saber por qué habían sucedido las reacciones ya descritas.

\section{* Tercera actividad}

En los informes de laboratorio se siguieron encontrando algunos errores menos significativos. En ocasiones, repetían el error de bajar la información de Internet sin siquiera leer $y$, ante esto, lo que se hacía era devolver el trabajo y que se realizara de forma correcta. En la redacción de las observaciones se empleó un lenguaje poco técnico, lo que resultó ser un aspecto un poco difícil de mejorar, ya que a pesar de que en los laboratorios se manejaban lenguajes técnicos, ellos reincidían. Por ejemplo insistían en que el olor del vino era "picho".

\section{* Cuarta y quinta actividad}

Al iniciar el proyecto, tal y como se mostró en el primer informe, solo dos estudiantes mos- 
traron actitud hacia la ciencia; para el final del proyecto fueron 7 estudiantes.

El análisis de estas actividades muestra cómo los estudiantes necesitan acompañamiento para corregir sus errores y que el método de ensayo y error es bastante plausible. En un principio los estudiantes mostraron rechazo, ya que consideraban que era demasiado trabajo, pero, desde mi punto de vista, fue ese trabajo constante el que los llevó a obtener los resultados esperados.

Por otro lado, con el laboratorio casero se logró el objetivo principal de este proyecto, el de interesar a los estudiantes por la Química. Así se demostró que la cantidad y variedad de preguntas que realizaron los estudiantes significan un importante factor en los procesos de investigación.

\section{Conclusiones}

- Los procesos de experimentación influyen positivamente en los procesos de aprendizaje y de desarrollo de actitud científica en los estudiantes.

- El método de ensayo y error resulta favorable para el aprendizaje de la elaboración de informes científicos, ya que para los estudiantes implican tiempo y un análisis detallado de los errores cometidos.

- Los estudiantes valoraron la importancia de recurrir a diferentes fuentes bibliográficas, debido a que no todos los autores abarcan los temas de igual manera y es importante conocer diferentes enfoques teóricos.

\section{Bibliografía}

Avery, C. S. (1991). Aprender cómo se investiga. Investigar cómo se aprende. En -Olson, M. (comp.) La investigación-acción entra al aula (pp.43-55). Argentina: Aique. Segunda edición.

Gil, D. (1993). Contribución de la historia y de la filosofía de las ciencias al desarrollo de un modelo de enseñanza/aprendizaje como investigación. Enseñanza de las ciencias.11, 197-212.

Tamayo, M. (1994). Metodología formal de la investigación científica. Bogotá: Grupo Noriega Editores. Segunda edición.

Tamayo, M. (1996). El proceso de la investigación científica. México: Grupo Noriega Editores. Tercera edición.

Sagan, C. (1995). El mundo y sus demonios. Barcelona: Planeta Editorial.

Williams, B. (1991). La ética y los límites de la filosofía. Venezuela: Monte Ávila Editores Latinoamericana, C.A. 